\title{
Reduzierte Erkrankungsaktivität im MRT bei günstigem Nebenwirkungsprofil
}

Fragestellung: Ist der selektive Sphingosin-1-Phosphat-Rezeptormodulator Ozanimod in der Therapie der schubförmigen Multiplen Sklerose (MS) wirksam?

Hintergrund: Mit Fingolimod wurde bereits 2011 der erste Sphingosin-1-Phosphat-Rezeptormodulator zur Behandlung der schubförmigen MS zugelassen und hat sich inzwischen in der Behandlung etabliert. In den zulassungsrelevanten Phase-IIIStudien zeigte Fingolimod hinsichtlich der jährlichen Schubrate sowohl gegen Placebo als auch gegen Interferon- $\beta$-1a jeweils eine Reduktion von 50-55\%. In der hier vorgestellten PhaseII-Studie wird nun ein weiterer selektiver Sphingosin-1-Phosphat-Rezeptormodulator hinsichtlich Sicherheit und Wirksamkeit in der Behandlung der schubförmigen MS überprüft.

Patienten und Methoden: Die RADIANCE-Studie wurde als kombinierte Phase-II-/III-Studie konzipiert. Beteiligt waren insgesamt 55 akademische und private MS-Zentren in 13 Ländern in Europa und den USA. Eingeschlossen wurden Patienten mit schubförmig verlaufender MS im Alter zwischen 18 und 55 Jahren, die einen EDSS-Score zwischen 0 und 5,0 sowie einen oder mehrere Schübe in den letzten zwölf Monaten erlit-

Cohen JA, Arnold DL, Cony G et al. Safety and efficacy of the selective sphingosine-1-phosphat receptor modulator ozanimod in relapsing multiple sclerosis (RADIANCE): A randomised placebo-controlled, phase-II trial. Lancet Neurol 2016: 15; $373-81$ ten hatten. Darüber hinaus mussten die Studienteilnehmer einen oder mehrere gadoliniumaufnehmende Herde in den vorangegangenen zwölf Monaten vor Screening aufweisen.

Die Randomisierung erfolgte computergesteuert $\mathrm{zu}$ gleichen Anteilen in die Be- handlungsgruppe mit Ozanimod 0,5 mg oder $1 \mathrm{mg}$ oder Placebo. Die Behandlungsdauer war auf 24 Wochen festgesetzt.

Um kardiologische Eindosierungseffekte bei der Erstgabe zu vermeiden, wurde Ozanimod in der ersten Woche von $0,25 \mathrm{mg}$ auf 0,5 mg oder auf $1 \mathrm{mg}$ über acht Tage aufdosiert.

Der primäre Endpunkt war die kumulative Zahl aller gadoliniumaufnehmenden Herde, die von einem unabhängigen MRTAnalysezentrum zwölf und 24 Wochen nach dem Beginn der Behandlung erhoben wurden.

Ergebnisse: Der erste Studienteilnehmer wurde im Oktober 2012 und der letzte Patient im Mai 2014 eingeschlossen. Die Intention-to-treat- und Safety-Population umfasste 258 Patienten. Davon wurden 88 Patienten mit Placebo, 87 mit Ozanimod 0,5 mg und 83 mit Ozanimod $1 \mathrm{mg}$ behandelt. Insgesamt $98 \%$ der Patienten beendeten die Behandlung.

Die mittlere kumulative Zahl der gadoliniumaufnehmenden Herde in den Wochen zwölf bis 24 betrug 11,1 in der Placebogruppe, 1,5 in der Ozanimod-0,5 mg-Gruppe und 1,5 in der Ozanimod-1 mg-Gruppe.

Die Nebenwirkungsraten waren gering. Es wurden weder schwere Infektionen noch kardiologische Nebenwirkungen berichtet. Auch traten keine Fälle von Makulaödemen auf. Die häufigsten Nebenwirkungen waren Nasopharyngitis, Kopfschmerzen und Harnwegsinfektionen.

Schlussfolgerungen: Ozanimod reduzierte signifikant die im MRT sichtbare Erkrankungsaktivität bei Patienten mit schubförmiger MS und zeigte ein günstiges Sicherheits- und Nebenwirkungsprofil.

\section{- Kommentar von Volker Limmroth, Köln}

\section{Warten auf die Ergebnisse der Phase-III-Studien}

\begin{abstract}
Mit Ozanimod ist nun ein weiterer selektiver $\mathrm{S}_{1} \mathrm{P}_{1}$-Rezeptormodulator untersucht worden, der offensichtlich eine hohe Wirksamkeit zeigt. Ob die Substanz wirksamer als das bereits zugelassene Fingolimod ist, kann aus den hier vorgestellten Daten jedoch nicht geschlossen werden. Auch das auf den ersten Blick etwas günstiger erscheinende Nebenwirkungsprofil muss noch in größeren Kohorten bestätigt werden, da Nebenwirkungen wie Makulaödeme erst nach der Behandlung mehrerer hundert Patienten auftraten und diese Patienten überdies spezifische Risikofaktoren wie einen Diabetes mellitus aufwiesen. Die Ergebnisse der bereits angelaufenen Phase-IIIStudien werden daher interessant sein, insbesondere wenn sich das günstigere Sicherheitsprofil bestätigen sollte.
\end{abstract}

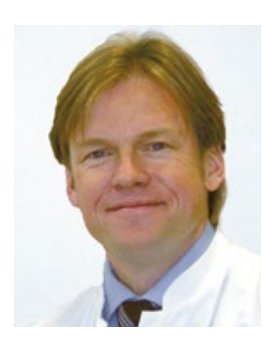

Prof. Dr. med. Volker Limmroth, Köln-Merheim

Chefarzt der Klinik für Neurologie und Palliativmedizin Köln-Merheim E-Mail: LimmrothV@kliniken-koeln.de 